Document downloaded from:

http://hdl.handle.net/10251/53460

This paper must be cited as:

González Estrada, OA.; Ródenas García, JJ.; Bordas, S.; Nadal, E.; Kerfriden, P.; Fuenmayor Fernández, FJ. (2015). Locally equilibrated stress recovery for goal oriented error estimation in the extended finite element method. Computers and Structures. 152:1-10. doi:10.1016/j.compstruc.2015.01.015.

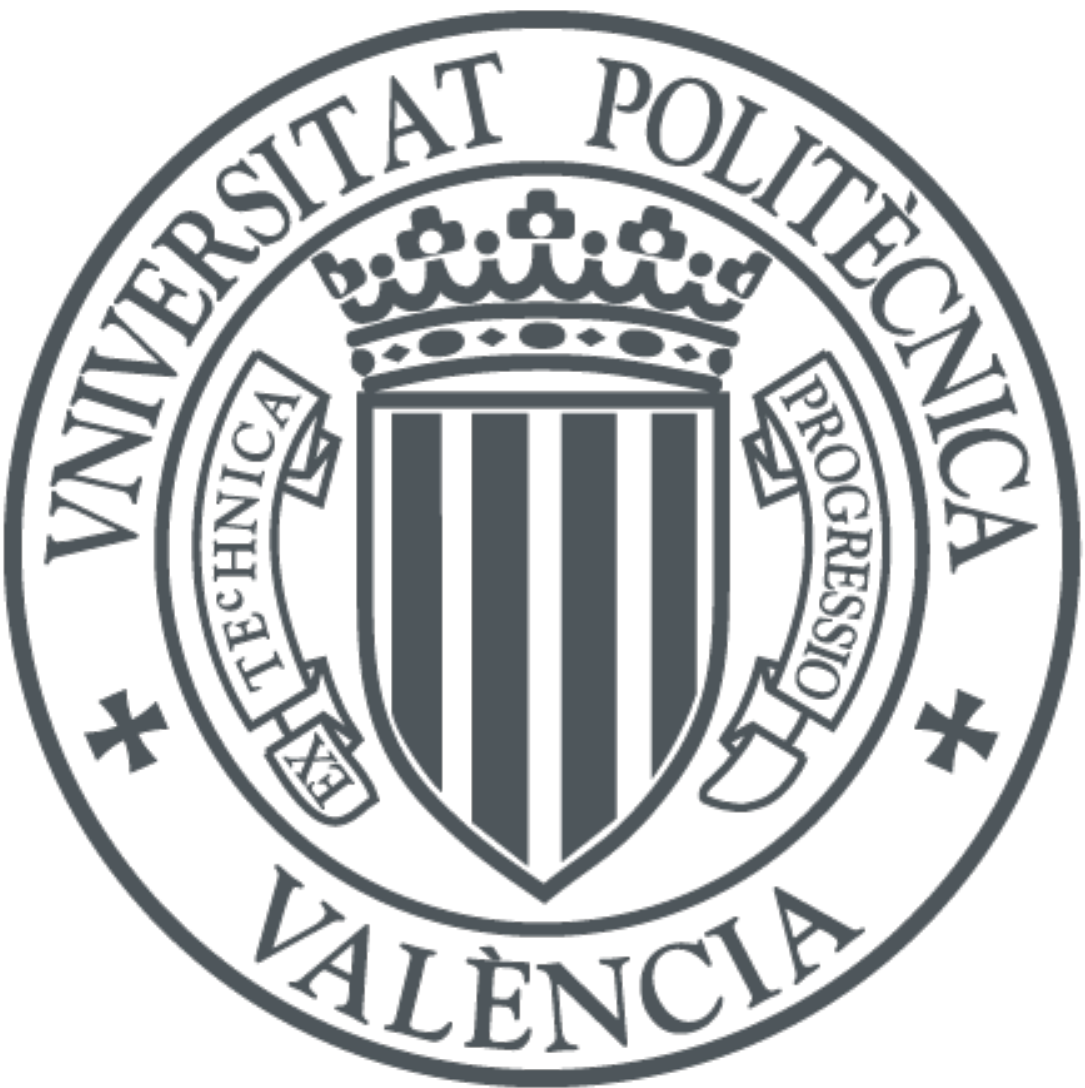

The final publication is available at

Copyright Elsevier

Additional Information 


\title{
Locally equilibrated stress recovery for goal oriented error estimation in the extended finite element method
}

\author{
O.A. González-Estrada ${ }^{1,3 *} \quad$ J.J. Ródenas ${ }^{2} \quad$ S.P.A. Bordas ${ }^{1}$ \\ E. Nadal ${ }^{2} \quad$ P. Kerfriden ${ }^{1} \quad$ F.J. Fuenmayor ${ }^{2}$
}

July 15,2015
${ }^{1}$ Institute of Mechanics and Advanced Materials (IMAM), Cardiff School of Engineering, Cardiff University, Queen's Buildings, The Parade, Cardiff CF24 3AA Wales, UK,
${ }^{2}$ Centro de Investigación de Tecnología de Vehículos (CITV),
Universitat Politècnica de València, E-46022-Valencia, Spain,
${ }^{3}$ School of Mechanical Engineering, Universidad Industrial de Santander, Bucaramanga, Colombia.

\begin{abstract}
Goal oriented error estimation and adaptive procedures are essential for the accurate and efficient evaluation of finite element numerical simulations that involve complex domains. By locally improving the approximation quality, for example, by using the extended finite element method (XFEM), we can solve expensive problems which could result intractable otherwise. Here, we present an error estimation technique for enriched finite element approximations that is based on an equilibrated recovery technique, which considers the stress intensity factor as the quantity of interest. The locally equilibrated superconvergent patch recovery is used to obtain enhanced stress fields for the primal and dual problems defined to evaluate the error estimate.
\end{abstract}

KEY WORDS: goal oriented, error estimation, recovery, quantities of interest, error control, mesh adaptivity

\section{Introduction}

In continuum mechanics, stresses and strains are usually the main quantities to describe the behaviour of a component under certain loads. However, when the component is affected by a crack these parameters are not sufficient to properly describe the behaviour of the component in the Linear Elasticity (LE) framework.

*Email: estradaoag@cardiff.ac.uk 
For instance, under the LE assumptions, the stress field at the crack tip will take infinite values, and in the surroundings of the crack tip, due to the high stress value, the small deformations assumption does not hold. Linear Elastic Fracture Mechanics (LEFM) assumptions are considered valid for brittle fracture [1,2]. One characterising parameter used to give a more realistic description of the behaviour around the crack tip is the stress intensity factor (SIF), which focuses in the local stress state at the crack tip [2] and can be considered an energy-based quantity. Hence, to properly describe the behaviour at the crack tip in LEFM is necessary to accurately evaluate the SIF. It results interesting to evaluate an error measure for the SIF to be able to control its level of accuracy [3, 4].

Since the beginning of the use of numerical simulations many methods have been developed to control the discretisation error of finite element approximations, mostly based on the evaluation of global error in energy norm of the Finite Element (FE) solution. These methods can be broadly classified in residual based [5], recovery based [6] and dual analysis [7, 8]. The numerical results in $[9,10,11]$ showed that a recovery technique with a standard superconvergent patch recovery (SPR) [12], applied in problems with smooth solution, was more robust than the residual estimates considered. However, a more interesting approach is to control the error in a particular quantity relevant for the design process $[13,14,15,16]$. This quantity could be defined as a bounded functional that describes the displacement or stresses in a given area of the domain, or for the case of fracture mechanics, the SIF that characterises the crack. This approach, referred to as goal oriented, is usually based on the use of duality techniques that involve the formulation of an adjoint or dual problem directly related to the quantity of interest (QoI). Residual methods have been frequently used to evaluate the error in quantities of interest although examples involving recovery techniques can be found in [17, 3], and considering dual analysis in [18]. In [19] an enhanced version of the SPR technique was used to obtain accurate estimations of the error in different QoI in the context of linear elasticity problems solved with the FEM. In [3], recovery and residual based estimates of the error in evaluating the $J$-integral (directly related to the SIF) for finite element (FE) approximations in the context of LEFM were presented. The authors showed that the most accurate error estimates for the J-integral were obtained with the recovery based estimators.

References $[16,15]$ showed that the error in the quantity of interest can be expressed in terms of errors in energy norm, and that if these errors in energy norm can be bounded we could also bound the error in the quantity of interest. On the other hand, it is usually difficult to obtain guaranteed error bounds of the quantities of interest while maintaining the accuracy of the estimate. The need of such a bound is also arguable in an engineering context as the reliability of an a posteriori error estimate, which is quantified by its local effectivity, can be verified beforehand on a number of practical cases. Here, we are interested in increasing the effectivity of the error estimate used to guide adaptive algorithms rather than error bounding.

In the context of LEFM, the extended finite element method (XFEM) [20] has been successfully used to enrich the finite element approximation in order to represent the particular features of cracks, namely, the discontinuity along the crack faces and the singularity at the crack tip. This method helps to overcome some of 
the difficulties when modelling crack propagation, such as the need for remeshing to obtain conforming meshes to the crack topology. Recent advanced numerical approaches for the purpose of XFEM and fracture analysis also include Mixed Discrete Least Squares Meshless (MDLSM) [21], and XIGA [22]. Error estimators in energy norm for XFEM and other partition of unity methods have been proposed in $[23,24,25,26]$ using recovery techniques, and in $[27,4,28]$ using the residual approach. A goal oriented approach for enriched finite element approximations based on the constitutive relation error has been presented in [29]. In [30] goal oriented error estimators based on the explicit residual method were introduce for the XFEM framework. In [31], adaptive techniques based on energy norm and goal oriented error estimation have been investigated for enriched finite element approximations.

In this paper, we propose a goal oriented error estimation technique for XFEM approximations that is based on the enhanced recovery technique previously presented in $[25,26]$ and the consideration of the SIF, typical of LEFM, as the quantity of interest. One of the key features of the recovery-based error estimators is that the solution is recovered patch-wise in a basis richer than the one used for the FE approximation. As shown in [23, 32], when XFEM is used, the basis used for the recovery should include the singular terms, which is not common in standard recovery techniques. Therefore, error estimates in quantities of interest will also require a careful consideration of the singular character of the XFEM solution, and the use of extended recovery approaches becomes a necessity to obtain accurate estimates. To improve the quality of the recovered stresses for the primal and dual problems, and therefore, the accuracy of the error estimate, we consider equilibrium constraints locally in patches of elements and the splitting of the recovered stress field into singular and smooth parts, which is the fundamental idea in the recovery process to describe the singular behaviour of the solution.

The paper is organised as follows. In Section 2, we introduce the problem under consideration and its corresponding enriched approximation. In Section 3, we show useful analytical definitions of QoI for the enforcement of equilibrium conditions. The recovery process is described in Section 4. We discuss the formulation of the dual problem when considering the stress intensity factor as the quantity of interest in the goal oriented approach. Numerical results are provided in Section 5 and conclusion are drawn in Section 6.

\section{Problem statement and XFEM for LEFM}

In this section, we introduce the 2D LEFM problem. We denote by $\mathbf{u}$ the displacement, by $\boldsymbol{\sigma}$ the Cauchy stress and by $\varepsilon$ the strain, all these fields defined over the domain $\Omega \subset \mathbb{R}^{2}$, of boundary denoted by $\partial \Omega . \Gamma_{N}$ and $\Gamma_{D}$ refer to the parts of the boundary where the Neumann and Dirichlet conditions are applied, and $\Gamma_{C}$ to the free traction surface describing a crack such that $\partial \Omega=\Gamma_{N} \cup \Gamma_{D} \cup \Gamma_{C}$ and $\Gamma_{N} \cap \Gamma_{D} \cap \Gamma_{C}=\emptyset$. We denote by $\mathbf{b}$ the body loads, $\mathbf{t}$ the tractions imposed along $\Gamma_{N}$ and $\boldsymbol{\sigma}_{0}, \boldsymbol{\varepsilon}_{0}$ the initial stresses and strains. The displacement field $\mathbf{u}$ is the solution 
of the problem given by

$$
\begin{aligned}
\mathbf{L}^{T} \boldsymbol{\sigma}+\mathbf{b} & =0 & & \text { in } \Omega, \\
\mathbf{G} \boldsymbol{\sigma} & =\mathbf{t} & & \text { on } \Gamma_{N}, \\
\mathbf{G} \boldsymbol{\sigma} & =\mathbf{0} & & \text { on } \Gamma_{C}, \\
\mathbf{u} & =\mathbf{0} & & \text { on } \Gamma_{D}, \\
\boldsymbol{\varepsilon}(\mathbf{u}) & =\mathbf{L} \mathbf{u} & & \text { in } \Omega, \\
\boldsymbol{\sigma} & =\mathbf{D}\left(\boldsymbol{\varepsilon}(\mathbf{u})-\boldsymbol{\varepsilon}_{0}\right)+\boldsymbol{\sigma}_{0} & & \text { in } \Omega,
\end{aligned}
$$

where $\mathbf{L}$ is the differential operator for linear elasticity, and $\mathbf{G}$ is the projection operator that projects the stress field into tractions over any boundary, with $\mathbf{n}$ the outward unit normal to $\Gamma_{N}$, such that

$$
\mathbf{L}^{T}=\left[\begin{array}{ccc}
\partial / \partial x & 0 & \partial / \partial y \\
0 & \partial / \partial y & \partial / \partial x
\end{array}\right], \quad \mathbf{G}=\left[\begin{array}{ccc}
n_{x} & 0 & n_{y} \\
0 & n_{y} & n_{x}
\end{array}\right]
$$

$\mathbf{D}$ is the matrix of the linear constitutive relation for stress and strain. We consider an homogeneous Dirichlet boundary condition in (4) for simplicity.

The problem expressed in its variational form is written as:

Find $\mathbf{u} \in V$ such that $\forall \mathbf{v} \in V=\left\{\mathbf{v}\left|\mathbf{v} \in\left[H^{1}(\Omega)\right]^{2}, \mathbf{v}\right|_{\Gamma_{D}}=\mathbf{0}\right\}$ :

$$
\int_{\Omega} \boldsymbol{\varepsilon}(\mathbf{u})^{T} \mathbf{D} \boldsymbol{\varepsilon}(\mathbf{v}) \mathrm{d} \Omega=\int_{\Omega} \mathbf{v}^{T} \mathbf{b} \mathrm{d} \Omega+\int_{\Gamma_{N}} \mathbf{v}^{T} \mathbf{t} \mathrm{d} \Gamma+\int_{\Omega} \boldsymbol{\varepsilon}(\mathbf{v})^{T} \mathbf{D} \varepsilon_{0} \mathrm{~d} \Omega-\int_{\Omega} \varepsilon^{T}(\mathbf{v}) \boldsymbol{\sigma}_{0} \mathrm{~d} \Omega .
$$

To evaluate the SIF, noted as $K$, it is common practice to use the interaction integral in its Equivalent Domain Integral (EDI) form. There are different expressions already available to evaluate EDI integrals for singular problems. In this work, we are going to consider the method based on extraction functions, as shown in [33]:

$$
K=-\frac{1}{C} \int_{\Omega_{I}} \boldsymbol{\sigma}^{T}\left[\begin{array}{c}
u_{x}^{\text {aux }} q_{, x} \\
u_{y}^{\text {aux }} q_{, y} \\
u_{y}^{\text {aux }} q_{, x}+u_{x}^{\text {aux }} q_{, y}
\end{array}\right]-\mathbf{u}^{T}\left[\begin{array}{c}
\sigma_{x x}^{\text {aux }} q_{, x}+\sigma_{x y}^{\text {aux }} q_{, y} \\
\sigma_{x y}^{\text {aux }} q_{, x}+\sigma_{y y}^{\text {aux }} q_{, y}
\end{array}\right] \mathrm{d} \Omega,
$$

where $\mathbf{u}^{\text {aux }}, \boldsymbol{\sigma}^{\text {aux }}$ are the auxiliary fields used to extract the SIFs in mode I or mode II and $C$ is a constant that is dependent on the geometry and the loading mode. $q$ is an arbitrary $C^{0}$ function that defines the extraction zone $\Omega_{I}$ which takes the value of 1 at the singular point and 0 at the boundary $\Gamma q_{, x}$ and $q_{, y}$ are the derivatives of the function $q$ with respect to $x$ and $y$.

\subsection{Discrete problem using XFEM}

Let us consider a finite element approximation of $\mathbf{u}$ denoted as $\mathbf{u}^{h}$. In the XFEM formulation [20], the approximation is usually enriched with two types of enrichment functions by means of the partition of unity: (i) a Heaviside function $H$ to describe the discontinuity of the displacement field along the crack, in the set of nodes $I^{\text {crack }}$ whose support is intersected by the crack and (ii) a set of branch functions $F_{\ell}$ to represent the asymptotic behaviour of the stress field near the crack tip, in the 
set of nodes $I^{\text {tip }}$ whose support contains the singularity. The XFEM displacement interpolation in a $2 \mathrm{D}$ model reads:

$$
\mathbf{u}^{h}(\mathbf{x})=\sum_{i \in I} N_{i}(\mathbf{x}) \mathbf{a}_{i}+\sum_{i \in I^{\text {crack }}} N_{i}(\mathbf{x}) H(\mathbf{x}) \mathbf{b}_{i}+\sum_{i \in I^{t i p}} N_{i}(\mathbf{x})\left(\sum_{\ell=1}^{4} F_{\ell}(\mathbf{x}) \mathbf{c}_{i}^{\ell}\right),
$$

where $N_{i}$ denotes the classical shape functions associated with node $i$ and $\mathbf{a}, \mathbf{b}, \mathbf{c}$ are the unknown coefficients. Note that we have four coefficients $\mathbf{c}^{\ell}$ multiplying each of the terms for the $F_{\ell}$ functions used in this paper for the $2 \mathrm{D}$ case, defined as [20]:

$$
\left\{F_{\ell}(r, \phi)\right\} \equiv \sqrt{r}\left\{\sin \frac{\phi}{2}, \cos \frac{\phi}{2}, \sin \frac{\phi}{2} \sin \phi, \cos \frac{\phi}{2} \sin \phi\right\} .
$$

Considering the enriched finite-dimensional subspace $V^{h} \subset V$ spanned by locally supported finite element shape functions, we solve for a discrete solution $\mathbf{u}^{h} \in V^{h}$ of the variational problem in (8) such that $\forall \mathbf{v} \in V^{h}$ :

$$
\begin{gathered}
\int_{\Omega} \boldsymbol{\varepsilon}\left(\mathbf{u}^{h}\right)^{T} \mathbf{D} \boldsymbol{\varepsilon}(\mathbf{v}) \mathrm{d} \Omega=\int_{\Omega} \boldsymbol{\sigma}^{T}\left(\mathbf{u}^{h}\right) \mathbf{D}^{-1} \boldsymbol{\sigma}(\mathbf{v}) \mathrm{d} \Omega= \\
\int_{\Omega} \mathbf{v}^{T} \mathbf{b} \mathrm{d} \Omega+\int_{\Gamma_{N}} \mathbf{v}^{T} \mathbf{t} \mathrm{d} \Gamma+\int_{\Omega} \boldsymbol{\varepsilon}(\mathbf{v})^{T} \mathbf{D} \boldsymbol{\varepsilon}_{0} \mathrm{~d} \Omega-\int_{\Omega} \boldsymbol{\varepsilon}(\mathbf{v})^{T} \boldsymbol{\sigma}_{0} \mathrm{~d} \Omega .
\end{gathered}
$$

Once we solve the XFEM problem formulated in (12), we can obtain an approximation to the exact value of the stress intensity factor $K^{*}$ using

$$
K^{*}=-\frac{1}{C} \int_{\Omega_{I}}\left(\boldsymbol{\sigma}^{h}\right)^{T}\left[\begin{array}{c}
u_{x}^{\text {aux }} q_{, x} \\
u_{y}^{\text {aux }} q_{, y} \\
u_{y}^{\text {aux }} q_{, x}+u_{x}^{\text {aux }} q_{, y}
\end{array}\right]-\left(\mathbf{u}^{h}\right)^{T}\left[\begin{array}{c}
\sigma_{x x}^{\text {aux }} q_{, x}+\sigma_{x y}^{\text {aux }} q_{, y} \\
\sigma_{x y}^{\text {aux }} q_{, x}+\sigma_{y y}^{\text {aux }} q_{, y}
\end{array}\right] \mathrm{d} \Omega,
$$

where $\boldsymbol{\sigma}^{h}=\mathbf{D}\left(\boldsymbol{\varepsilon}\left(\mathbf{u}^{h}\right)-\boldsymbol{\varepsilon}_{0}\right)+\boldsymbol{\sigma}_{0}$ is the XFEM stress solution.

\section{Error in quantities of interest}

Traditionally, the discretisation error, defined as $\mathbf{e}:=\mathbf{u}-\mathbf{u}^{h}$ in the absence of other types of errors, is measured as the error in energy norm. Now we will show the expressions used to evaluate the error in energy norm which will serve to introduce the error estimation in Quantities of Interest in this section.

To quantify the error introduced by the discretisation a common approach is to use the energy norm of e defined as:

$$
\|\mathbf{e}\|^{2}=\int_{\Omega} \boldsymbol{\varepsilon}(\mathbf{e})^{T} \mathbf{D} \boldsymbol{\varepsilon}(\mathbf{e}) \mathrm{d} \Omega .
$$

Using the constitutive relation and introducing the error in the stress field $\mathbf{e}_{\sigma}:=$ $\boldsymbol{\sigma}-\boldsymbol{\sigma}^{h}$ the previous expression can be written as:

$$
\|\mathbf{e}\|^{2}=\int_{\Omega} \mathbf{e}_{\sigma}^{T} \mathbf{D}^{-1} \mathbf{e}_{\sigma} \mathrm{d} \Omega
$$


Whereas the exact field $\mathbf{u}$ is in general unknown, it is possible to obtain an estimate of the error by means of the approximation introduced in [6] in the context of $\mathrm{FE}$ elasticity problems:

$$
\|\mathbf{e}\|^{2} \approx \int_{\Omega}\left(\mathbf{e}_{\sigma}^{*}\right)^{T} \mathbf{D}^{-1}\left(\mathbf{e}_{\sigma}^{*}\right) \mathrm{d} \Omega
$$

where $\mathbf{e}_{\sigma}^{*}$ is the approximated stress error defined by $\mathbf{e}_{\sigma}^{*}:=\boldsymbol{\sigma}^{*}-\boldsymbol{\sigma}^{h}$, being $\boldsymbol{\sigma}^{*}$ the recovered stress field. Local element contributions are also obtained from (16) considering the domain of the element $\Omega_{e}$.

\subsection{Error estimation in QoI}

The goal of many numerical computations is to control a specific design parameter, thus, it results natural to formulate the error in terms of such quantity instead of in energy norm. In this case, as indicated in section 2, the quantity that describes the behaviour of a component in the surroundings of the crack tip is the SIF, then we are interested in controlling the accuracy of the SIF instead of traditional measures such as the error in energy norm. For this purpose, error estimators measured in the energy norm might be utilised to estimate the error in a particular quantity of interest [13]. In this section we show how to a modified version of the Zienkiewicz and Zhu (ZZ) error estimator presented in [6] is used with the SPR-CX recovery to evaluate the error in quantities of interest.

A common approach to evaluate the error in QoI involves the use of duality techniques which solve two different problems. A primal problem, which is the problem at hand as shown in (8), and a dual problem used to extract information on the QoI. Thus, we shall explain the formulation of the dual problem.

Consider the primal problem given in (8) and its approximate finite element solution $\mathbf{u}^{h} \in V^{h} \subset V$. Let $Q: V \rightarrow \mathbb{R}$ be a bounded linear functional representing some quantity of interest, acting on the space $V$ of admissible functions for the problem at hand. We are interested in estimating the error in the functional $Q(\mathbf{u})$ when calculated using the value of the approximate solution $\mathbf{u}^{h}$ :

$$
Q(\mathbf{u})-Q\left(\mathbf{u}^{h}\right)=Q\left(\mathbf{u}-\mathbf{u}^{h}\right)=Q(\mathbf{e}) .
$$

To evaluate $Q(\mathbf{e})$, the standard procedure is to solve the auxiliary or dual problem

$$
\begin{aligned}
& \text { Find } \tilde{\mathbf{u}} \in V \text { such that } \forall \mathbf{v} \in V, \\
& \int_{\Omega} \boldsymbol{\varepsilon}(\mathbf{v})^{T} \mathbf{D} \boldsymbol{\varepsilon}(\tilde{\mathbf{u}}) \mathrm{d} \Omega=\mathrm{Q}(\mathbf{v}),
\end{aligned}
$$

which can be seen as the variational form of an auxiliary mechanical problem used to extract information of the QoI. The dual displacement field $\tilde{\mathbf{u}} \in V$ vanishes over $\Gamma_{D}$. Test function $\mathbf{v}$ is a virtual displacement. Field $\tilde{\boldsymbol{\sigma}}=\mathbf{D}\left(\varepsilon(\tilde{\mathbf{u}})-\tilde{\boldsymbol{\varepsilon}}_{0}\right)+\tilde{\boldsymbol{\sigma}}_{0}$, where $\tilde{\boldsymbol{\sigma}}_{0}$ and $\tilde{\boldsymbol{\varepsilon}}_{0}$ are known initial stress and strain, can be interpreted as a mechanical stress field. The left-hand side of (18) is the work of internal forces of the auxiliary mechanical problem and $Q(\mathbf{v})$ is the work of an abstract external load. 
We consider the same finite element space used in the primal problem to look for an approximation of $\tilde{\mathbf{u}} \in V$ such that the problem is

$$
\begin{aligned}
& \text { Find } \tilde{\mathbf{u}}^{h} \in V^{h} \text { such that } \forall \mathbf{v} \in V^{h}, \\
& \int_{\Omega} \boldsymbol{\varepsilon}(\mathbf{v})^{T} \mathbf{D} \boldsymbol{\varepsilon}\left(\tilde{\mathbf{u}}^{h}\right) \mathrm{d} \Omega=\mathrm{Q}(\mathbf{v}) .
\end{aligned}
$$

To obtain an exact representation for the error $Q(\mathbf{e})$ in terms of the solution of the dual problem we substitute $\mathbf{v}=\mathbf{e}$ in (18) and, considering the Galerkin orthogonality, for all $\tilde{\mathbf{u}}^{h} \in V^{h}$ :

$$
Q(\mathbf{e})=\int_{\Omega} \boldsymbol{\varepsilon}(\mathbf{e})^{T} \mathbf{D} \boldsymbol{\varepsilon}(\tilde{\mathbf{e}}) \mathrm{d} \Omega
$$

where $\tilde{\mathbf{e}}:=\tilde{\mathbf{u}}-\tilde{\mathbf{u}}^{h}$ is the discretisation error of the dual problem (18). We can obtain an expression in terms of the mechanical stresses using the constitutive relation:

$$
Q(\mathbf{e})=\int_{\Omega} \mathbf{e}_{\sigma}^{T} \mathbf{D}^{-1} \tilde{\mathbf{e}}_{\boldsymbol{\sigma}} \mathrm{d} \Omega
$$

where $\tilde{\mathbf{e}}_{\sigma}:=\tilde{\boldsymbol{\sigma}}-\tilde{\boldsymbol{\sigma}}^{h}$ is the stress error of the dual problem and $\tilde{\boldsymbol{\sigma}}^{h}=\mathbf{D}\left(\varepsilon\left(\tilde{\mathbf{u}}^{h}\right)-\right.$ $\left.\tilde{\boldsymbol{\varepsilon}}_{0}\right)+\tilde{\boldsymbol{\sigma}}_{0}$ the finite element stress field.

\subsection{Recovery-based error estimate in QoI}

The error in the QoI in (21) is related to the errors in the FE approximations $\mathbf{u}^{h}$ and $\tilde{\mathbf{u}}^{h}$. Thus, we can select from the set of available procedures to estimate the error in the energy norm a technique to obtain estimates of the error in the QoI. Considering expressions (16) and (21) we can derive an estimate for the error in the QoI which reads

$$
Q(\mathbf{e}) \approx \mathcal{E}=\int_{\Omega}\left(\mathbf{e}_{\boldsymbol{\sigma}}^{*}\right)^{T} \mathbf{D}^{-1}\left(\tilde{\mathbf{e}}_{\boldsymbol{\sigma}}^{*}\right) \mathrm{d} \Omega,
$$

where the approximate dual error is $\tilde{\mathbf{e}}_{\sigma}^{*}=\tilde{\boldsymbol{\sigma}}^{*}-\tilde{\boldsymbol{\sigma}}^{h}$ and $\tilde{\boldsymbol{\sigma}}^{*}$ is the recovered auxiliary stress field. Here, we expect to have a sharp estimate of the error in the QoI if the recovered stress fields are accurate approximations to their exact counterparts.

The recovered stress fields can be computed in many ways, for example, by using the SPR technique as explained in [12]. To obtain accurate representations of the exact stress fields for the primal and dual solutions, we propose the use of the locally equilibrated recovery technique described in Section 4. This technique, which is an enhancement of the SPR, enforces the fulfilment of the internal and boundary equilibrium equations locally on patches. For problems with singularities the stress field is also decomposed into two parts: smooth and singular, which are separately recovered.

Two remarks have to be made. First, the analytical expressions that define the loads for the dual problem are obtained from the interpretation of the functional $Q$ in terms of tractions, body loads, initial stresses and strains. Second, to enforce equilibrium conditions during the recovery process along the boundary of the domain of interest (DoI) used to define the QoI, we consider it as an internal interface. We 
use different polynomial expansions on each side of the boundary and enforce statical admissibility of the normal and tangential stresses as it will be explained in Section 4

\subsection{Analytical definition of the dual problem}

The recovery technique presented in [25] and used in this contribution requires that the mechanical equilibrium must be made explicit in order to recover the dual stress field. Thus, the right-hand side of (18) is interpreted as the work of mechanical external forces, and the analytical expression of these forces is derived, depending on the quantity of interest:

Find $\tilde{\mathbf{u}} \in V$ such that $\forall \mathbf{v} \in V$ :

$$
\begin{aligned}
& \int_{\Omega} \boldsymbol{\varepsilon}(\mathbf{v})^{T} \mathbf{D} \boldsymbol{\varepsilon}(\tilde{\mathbf{u}}) \mathrm{d} \Omega=Q(\mathbf{v}) \\
& =\int_{\Omega} \mathbf{v}^{T} \tilde{\mathbf{b}} \mathrm{d} \Omega+\int_{\Gamma_{N}} \mathbf{v}^{T} \tilde{\mathbf{t}} \mathrm{d} \Gamma+\int_{\Omega} \boldsymbol{\varepsilon}(\mathbf{v})^{T} \mathbf{D} \tilde{\boldsymbol{\varepsilon}}_{0} \mathrm{~d} \Omega-\int_{\Omega} \boldsymbol{\varepsilon}(\mathbf{v})^{T} \tilde{\boldsymbol{\sigma}}_{0} \mathrm{~d} \Omega .
\end{aligned}
$$

The problem in (23) is solved using a FE approximation with test and trial functions in $V^{h}$. The finite element solution is denoted by $\tilde{\mathbf{u}}^{h} \in V^{h}$.

Such derivations were presented in $[34,35,36]$. Here, we only recall the one that is of interest in LEFM, the SIF. In this case, $Q(\mathbf{u})=K$. Equation (9) can be written as:

$$
\begin{array}{r}
Q(\mathbf{u})=K=\int_{\Omega_{I}}(\boldsymbol{\sigma})^{T}\left(-\frac{1}{C}\right)\left[\begin{array}{c}
u_{x}^{\text {aux }} q_{, x} \\
u_{y}^{\text {aux }} q_{, y} \\
u_{u}^{\text {aux }} q_{, x}+u_{x}^{\text {aux }} q_{, y}
\end{array}\right]- \\
(\mathbf{u})^{T}\left(-\frac{1}{C}\right)\left[\begin{array}{l}
\sigma_{x x}^{\text {aux }} q_{, x}+\sigma_{x y}^{\text {aux }} q_{, y} \\
\sigma_{x y}^{\text {aux }} q_{, x}+\sigma_{y y}^{\text {aux }} q_{, y}
\end{array}\right] \mathrm{d} \Omega .
\end{array}
$$

Comparing (23) with (24) we can rewrite (24) as a function of initial strains $\tilde{\varepsilon}_{0}$ and body loads $\tilde{\mathbf{b}}$ :

$$
Q(\mathbf{u})=K=\int_{\Omega_{I}} \boldsymbol{\sigma}(\mathbf{u})^{T} \tilde{\varepsilon}_{0}+(\mathbf{u})^{T} \tilde{\mathbf{b}} \mathrm{d} \Omega .
$$

Thus, if we replace $\mathbf{u}$ with the vector of arbitrary displacements $\mathbf{v}$, the quantity of interest can be evaluated from

$$
Q(\mathbf{v})=\int_{\Omega_{I}} \boldsymbol{\sigma}(\mathbf{v})^{T} \tilde{\varepsilon}_{0} \mathrm{~d} \Omega+\int_{\Omega_{\mathrm{I}}} \mathbf{v}^{\mathrm{T}} \tilde{\mathbf{b}} \mathrm{d} \Omega .
$$

Hence, the initial strains and the body loads per unit volume that can be applied in the dual problem to extract the SIF are

$$
\tilde{\varepsilon}_{0}=-\frac{1}{C}\left[\begin{array}{c}
u_{1}^{\text {aux }} q_{, 1} \\
u_{2}^{\text {aux }} q_{, 2} \\
u_{2}^{\text {aux }} q_{, 1}+u_{1}^{\text {aux }} q_{, 2}
\end{array}\right] \quad, \quad \tilde{\mathbf{b}}=\frac{1}{C}\left[\begin{array}{c}
\sigma_{11}^{\text {aux }} q_{, 1}+\sigma_{21}^{\text {aux }} q_{, 2} \\
\sigma_{12}^{\text {aux }} q_{, 1}+\sigma_{22}^{\text {aux }} q_{, 2}
\end{array}\right] .
$$




\section{The recovery technique}

In this work we consider the SPR-CX recovery technique, introduced in [25], which is an enhancement of the error estimator introduced in [37], to recover the solutions for the primal and dual problems. The technique incorporates the ideas in [38] to guarantee locally on patches the exact satisfaction of the equilibrium equations, and the extension in [25] to singular problems.

Let us define the field $\sigma^{-h}$ such that we subtract the initial stress and strain from the field $\boldsymbol{\sigma}^{h}$ :

$$
\boldsymbol{\sigma}^{-h}=\boldsymbol{\sigma}^{h}-\boldsymbol{\sigma}_{0}+\mathbf{D} \varepsilon_{\mathbf{0}},
$$

and perform the recovery on $\boldsymbol{\sigma}^{-h}$. Then, the recovered field is

$$
\sigma^{*}=\sigma^{-*}+\sigma_{0}-\mathrm{D} \varepsilon_{0},
$$

where $\boldsymbol{\sigma}^{-*}$ is the recovered field that corresponds to $\boldsymbol{\sigma}^{-h}$.

\subsection{Splitting concept}

Different techniques have been used to account for the singular part during the recovery process $[23,25]$. Here, following the ideas in [25], for singular problems we can consider that the exact stress field $\boldsymbol{\sigma}$ allows to be decomposed into two stress fields, a smooth field $\boldsymbol{\sigma}_{\text {smo }}$ and a singular field $\boldsymbol{\sigma}_{\text {sing }}$ :

$$
\sigma=\sigma_{\text {smo }}+\sigma_{\text {sing }} .
$$

This idea is to decompose the XFEM stress field $\boldsymbol{\sigma}^{-h}$ into two parts, one smooth $\boldsymbol{\sigma}_{\text {smo }}^{-h}$ and another singular $\boldsymbol{\sigma}_{\text {sing }}^{-h}$. The SPR-CX technique will use a different recovery procedure for each one of these parts. The purpose of this splitting is to account for the singular part of the stress field in the recovery process, which is necessary in the XFEM framework.

For the recovery of the singular part, the expressions which describe the asymptotic fields near the crack tip are used. To evaluate $\boldsymbol{\sigma}_{\text {sing }}^{-*}$ we first obtain estimated values of the stress intensity factors $K_{\mathrm{I}}^{*}$ and $K_{\mathrm{II}}^{*}$ using a domain integral (13) method based on extraction functions $[33,39]$. Notice that the recovered part $\boldsymbol{\sigma}_{\text {sing }}^{-*}$ is an equilibrated field as it satisfies the equilibrium equations.

Once the field $\boldsymbol{\sigma}_{\text {sing }}^{-*}$ has been evaluated, an FE-type approximation (discontinuous) to the smooth part $\boldsymbol{\sigma}_{\text {smo }}^{-h}$ can be obtained subtracting $\boldsymbol{\sigma}_{\text {sing }}^{-*}$ from the raw FE field:

$$
\boldsymbol{\sigma}_{\mathrm{smo}}^{-h}=\boldsymbol{\sigma}^{-h}-\boldsymbol{\sigma}_{\text {sing }}^{-*} .
$$

Then, the recovered field of the smooth part, $\boldsymbol{\sigma}_{\mathrm{smo}}^{-*}$, is evaluated applying the enhancements of the SPR technique presented in [38] to $\boldsymbol{\sigma}_{\mathrm{smo}}^{-h}$. Finally, the recovered stress field can be evaluated with the expression:

$$
\boldsymbol{\sigma}^{-*}=\boldsymbol{\sigma}_{\mathrm{smo}}^{-*}+\boldsymbol{\sigma}_{\mathrm{sing}}^{-*} .
$$


The concept of stress splitting and the recovery of the singular and smooth parts is used locally on patches, as described next in Section 4.2.

\subsection{Recovery of the smooth stress field}

The SPR-C (C stand for constraints) is a SPR-based stress recovery process that introduces a set of constraints to fulfil at each patch of elements equilibrium and compatibility conditions. This technique will be applied to the smooth part of the stress field $\boldsymbol{\sigma}_{\text {smo }}^{-h}$.

In the SPR-C technique, as in the original SPR technique, we define a patch $\mathcal{P}^{(J)}$ as the set of elements connected to a vertex node $J$. On each patch, a polynomial expansion for each one of the components of the recovered stress field is expressed in the form:

$$
\hat{\sigma}_{k}^{-*}(\mathbf{x})=\mathbf{p}(\mathbf{x}) \mathbf{a}_{k} \quad k=x x, y y, x y,
$$

where $\mathbf{p}$ represents a polynomial basis and $\mathbf{a}_{k}$ are unknown coefficients. Usually, the polynomial basis is chosen equal to the non-extended finite element basis for the displacements. A least squares approximation to the values of $\boldsymbol{\sigma}_{\mathrm{smo}}^{-h}$ evaluated at the integration points of the elements within the patch, $\mathbf{x}_{G} \in \mathcal{P}^{(J)}$, is used to evaluate the coefficients $\mathbf{a}_{k}$.

For the 2D case, the recovered stress field coupling the three stress components reads:

$$
\hat{\boldsymbol{\sigma}}_{s m o}^{-*}(\mathbf{x})=\left\{\begin{array}{l}
\hat{\sigma}_{x x}^{-*}(\mathbf{x}) \\
\hat{\sigma}_{y y}^{-*}(\mathbf{x}) \\
\hat{\sigma}_{x y}^{-*}(\mathbf{x})
\end{array}\right\}=\mathbf{P}(\mathbf{x}) \mathbf{A}=\left[\begin{array}{ccc}
\mathbf{p}(\mathbf{x}) & \mathbf{0} & \mathbf{0} \\
\mathbf{0} & \mathbf{p}(\mathbf{x}) & \mathbf{0} \\
\mathbf{0} & \mathbf{0} & \mathbf{p}(\mathbf{x})
\end{array}\right]\left\{\begin{array}{l}
\mathbf{a}_{x x} \\
\mathbf{a}_{y y} \\
\mathbf{a}_{x y}
\end{array}\right\}
$$

Note that in contrast to the basic SPR, the SPR-C technique uses a continuous least squares approach to obtain the coefficients $\mathbf{A}$, according the functional

$$
\mathcal{F}^{(J)}(A)=\int_{\mathcal{P}^{(J)}}\left(\mathbf{P A}-\boldsymbol{\sigma}_{\text {smo }}^{-h}\right)^{2} \mathrm{~d} \Omega
$$

\subsubsection{Equilibrium conditions}

Constraint equations are introduced via Lagrange multipliers into the functional defined in (35) on each patch, in order to enforce the satisfaction of the:

- Internal equilibrium equation: The constraint equation for the internal equilibrium in the patch is defined as:

$$
\forall \mathbf{x}_{\mathbf{j}} \in \mathcal{P}^{(J)} \quad \mathbf{L}^{T} \hat{\boldsymbol{\sigma}}_{\mathrm{smo}}^{-*(J)}\left(\mathbf{x}_{j}\right)+\mathbf{L}^{T}\left(\boldsymbol{\sigma}_{0}\left(\mathbf{x}_{j}\right)-\mathbf{D} \boldsymbol{\varepsilon}_{0}\left(\mathbf{x}_{j}\right)\right)+\hat{\mathbf{b}}\left(\mathbf{x}_{j}\right):=\mathbf{c}^{\mathrm{int}}\left(\mathbf{x}_{j}\right)=0
$$

where $\hat{\mathbf{b}}(\mathbf{x})$ is a polynomial least squares fit of degree $p-1$ to the actual body forces $\mathbf{b}(\mathbf{x})$, being $p$ the degree of the recovered stress field $\hat{\boldsymbol{\sigma}}_{\text {smo }}^{-*(J)}$. We enforce $\mathbf{c}^{\text {int }}\left(\mathbf{x}_{j}\right)$ at a sufficient number of $j$ non-aligned points (nie) to guarantee the exact representation of $\hat{\mathbf{b}}(\mathbf{x})$, e.g. three points for a linear representation. This procedure will add one equation per point $j$ to the linear system to solve at each patch. 
- Boundary equilibrium equations: We use a point collocation approach to impose the satisfaction of a polynomial approximation to the tractions along the Neumann boundary intersecting the patch. The constraint equation reads

$$
\begin{aligned}
& \forall \mathbf{x}_{j} \in \Gamma_{N} \cap \mathcal{P}^{(J)} \\
& \mathbf{G} \hat{\boldsymbol{\sigma}}_{\text {smo }}^{-*(J)}\left(\mathbf{x}_{j}\right)+\mathbf{G} \mathbf{L}^{T}\left(\boldsymbol{\sigma}_{0}\left(\mathbf{x}_{j}\right)-\mathbf{D} \varepsilon_{0}\left(\mathbf{x}_{j}\right)\right)+\mathbf{G} \boldsymbol{\sigma}_{\text {sing }}^{-*}-\mathbf{t}\left(\mathbf{x}_{j}\right):=\mathbf{c}^{\operatorname{ext}}\left(\mathbf{x}_{j}\right)=0 .
\end{aligned}
$$

We enforce $\mathbf{c}^{\text {ext }}\left(\mathbf{x}_{j}\right)$ in $n b e=p+1$ points along the part of the boundary crossing the patch. In the case that more than one boundary intersects the patch, only one curve is considered in order to avoid over-constraining.

- Compatibility equations: $\mathbf{c}^{\mathrm{cmp}}\left(\mathbf{x}_{j}\right)$ is only imposed in the case that $p \geq 2$ in a sufficient number of non-aligned points. $\hat{\boldsymbol{\sigma}}_{\text {smo }}^{-*}$ directly satisfies $\mathbf{c}^{c m p}$ for $p=1$.

Thus, the Lagrange functional enforcing the constraint equations for a patch $\mathcal{P}^{(J)}$ can be written as

$\mathcal{L}^{(J)}(\mathbf{A}, \boldsymbol{\lambda})=\mathcal{F}^{(J)}(\mathbf{A})+\sum_{i=1}^{n i e} \lambda_{i}^{\mathrm{int}}\left(\mathbf{c}^{\mathrm{int}}\left(\mathbf{x}_{i}\right)\right)+\sum_{j=1}^{n b e} \lambda_{j}^{\mathrm{ext}}\left(\mathbf{c}^{\mathrm{ext}}\left(\mathbf{x}_{j}\right)\right)+\sum_{k=1}^{n c} \lambda_{k}^{\mathrm{cmp}}\left(\mathbf{c}^{\mathrm{cmp}}\left(\mathbf{x}_{k}\right)\right)$.

This functional will expand the linear system to solve at each patch to include the additional equations to enforce the internal and boundary equilibrium and the compatibility equation.

Optimizing functional (38) we obtain a linear system of equations to evaluate the coefficients A. To enforce equilibrium conditions along internal boundaries (e.g. bimaterial problems, problems with zones subjected to different body forces, etc.), we consider different polynomial expansions on each side of the boundary and enforce the statical admissibility condition imposing equilibrium along this boundary. Suppose that we have a patch intersected by $\Gamma_{I}$ such that $\Omega_{e}=\Omega_{1, e} \cup \Omega_{2, e}$ for intersected elements, as shown in Figure 1. To enforce equilibrium conditions along $\Gamma_{I}$ we define the stresses $\hat{\boldsymbol{\sigma}}_{\Omega_{1}}^{-*}, \hat{\boldsymbol{\sigma}}_{\Omega_{2}}^{-*}$ at each side of the internal boundary. Then, the boundary equilibrium along $\Gamma_{I}$ given the prescribed tractions $\mathbf{t}_{\Gamma_{I}}=\left[t_{x} t_{y}\right]^{T}$ is:

$$
\mathbf{G}\left(\left.\hat{\boldsymbol{\sigma}}_{\Omega_{1}}^{-*}\right|_{\Gamma_{I}}-\left.\hat{\boldsymbol{\sigma}}_{\Omega_{2}}^{-*}\right|_{\Gamma_{I}}\right)=\mathbf{t}_{\Gamma_{I}}
$$

The same procedure can be used for patches intersected by the crack. In this case, we could consider the traction-free condition along the crack faces or define a different prescribed condition depending on the configuration.

The continuity of the recovered field is obtained by using a partition of unity procedure [40] to weight the stress fields obtained from the patches formed at the vertex nodes of the element. The field $\boldsymbol{\sigma}^{-*}$ is interpolated using linear shape functions $N^{(J)}$ associated with the $n_{v}$ vertex nodes and adding the contributions of the smooth and singular parts as in (32), such that

$$
\boldsymbol{\sigma}^{-*}(\mathbf{x})=\sum_{J=1}^{n_{v}} N^{(J)}(\mathbf{x})\left(\hat{\boldsymbol{\sigma}}_{\text {smo }}^{-*(J)}(\mathbf{x})+\hat{\boldsymbol{\sigma}}_{\text {sing }}^{-*(J)}(\mathbf{x})\right) .
$$




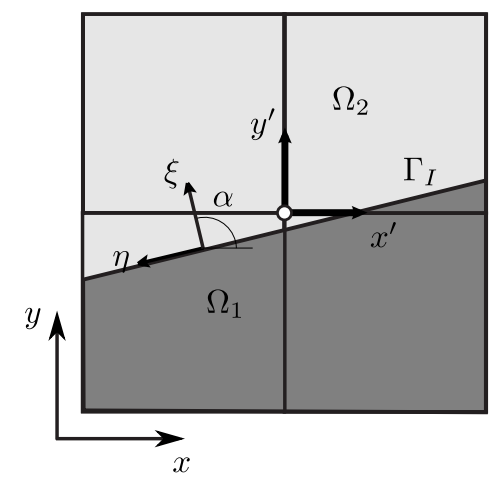

Figure 1: Equilibrium conditions along internal boundaries.

Here, to reduce the computational cost, $\hat{\boldsymbol{\sigma}}_{\text {sing }}^{-*}$ is evaluated over the set of points near the area of influence of the singularity, defined by the splitting radius, as shown in [25]. After evaluating the equilibrated recovered fields on each patch $\hat{\boldsymbol{\sigma}}^{-*(J)}$, we use (40) to obtain a continuous field. This process introduces a lack of equilibrium $\mathbf{s}=\sum_{J=1}^{n_{v}} \nabla N^{(J)} \hat{\boldsymbol{\sigma}}^{-*(J)}$ when evaluating the divergence of the internal equilibrium equation, as explained in [37, 26].

\section{$5 \quad$ Numerical results}

In this section we consider numerical examples for 2D problems with exact analytical solution to evaluate the performance of the technique presented above. For that purpose we define the effectivity index of the error estimator $\theta$ as:

$$
\theta=\frac{\mathcal{E}}{Q(\mathbf{e})}
$$

where $Q(\mathbf{e})$ denotes the exact error in the quantity of interest, and $\mathcal{E}$ represents the evaluated error estimate. We can also represent the effectivity in the QoI defined as

$$
\theta_{Q o I}=\frac{Q\left(\mathbf{u}^{h}\right)+\mathcal{E}}{Q(\mathbf{u})},
$$

and the relative error in the QoI for the exact and estimated error

$$
\eta^{Q}(\mathbf{e})=\frac{|Q(\mathbf{e})|}{|Q(\mathbf{u})|}, \quad \eta^{\mathcal{E}}=\frac{|\mathcal{E}|}{\left|Q\left(\mathbf{u}^{h}\right)+\mathcal{E}\right|} .
$$

\subsection{Westergaard problem - FEM solution.}

Let us consider the Westergaard problem [25, 41] of linear elastic fracture mechanics for which the exact analytical solution is known. The Westergaard problem corresponds to an infinite plate loaded at infinity with biaxial tractions $\sigma_{x \infty}=\sigma_{y \infty}=\sigma_{\infty}$ and shear traction $\tau_{\infty}$, presenting a crack of length $2 a$ as shown in Figure 2. Combining the externally applied loads we can obtain different loading conditions: pure mode I, pure mode II or mixed mode. 


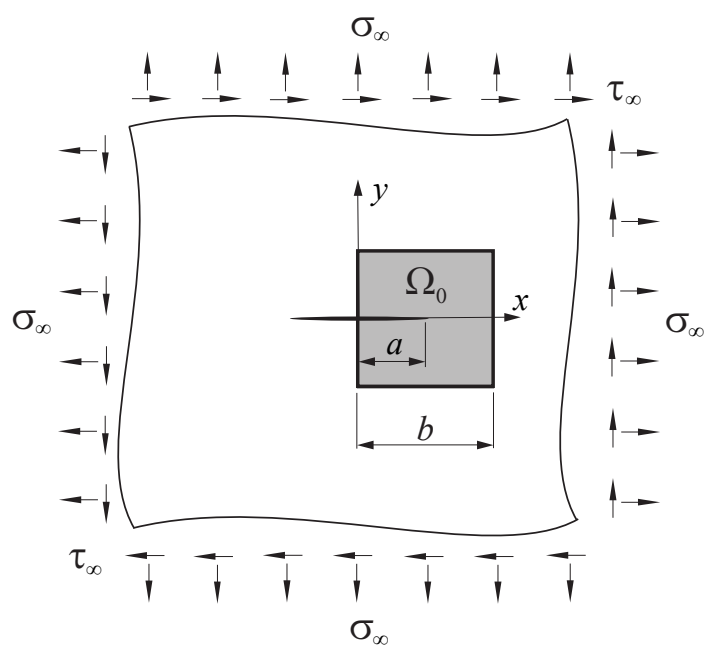

Figure 2: Westergaard problem. Infinite plate with a crack of length $2 a$ under uniform tractions $\sigma_{\infty}$ (biaxial) and $\tau_{\infty}$. Finite portion of the domain $\Omega_{0}$, modelled with FE.

The numerical model corresponds to a finite portion of the domain ( $a=5$ and $b=10$ in Figure 2). The applied projected stresses for mode I are evaluated from the analytical Westergaard solution [41]:

$$
\begin{aligned}
& \sigma_{x}^{I}(x, y)=\frac{\sigma_{\infty}}{\sqrt{|t|}}\left[\left(x \cos \frac{\phi}{2}-y \sin \frac{\phi}{2}\right)+y \frac{a^{2}}{|t|^{2}}\left(m \sin \frac{\phi}{2}-n \cos \frac{\phi}{2}\right)\right], \\
& \sigma_{y}^{I}(x, y)=\frac{\sigma_{\infty}}{\sqrt{|t|}}\left[\left(x \cos \frac{\phi}{2}-y \sin \frac{\phi}{2}\right)-y \frac{a^{2}}{|t|^{2}}\left(m \sin \frac{\phi}{2}-n \cos \frac{\phi}{2}\right)\right], \\
& \tau_{x y}^{I}(x, y)=y \frac{a^{2} \sigma_{\infty}}{|t|^{2} \sqrt{|t|}}\left(m \cos \frac{\phi}{2}+n \sin \frac{\phi}{2}\right)
\end{aligned}
$$

and for mode II:

$$
\begin{aligned}
& \sigma_{x}^{I I}(x, y)=\frac{\tau_{\infty}}{\sqrt{|t|}}\left[2\left(y \cos \frac{\phi}{2}+x \sin \frac{\phi}{2}\right)-y \frac{a^{2}}{|t|^{2}}\left(m \cos \frac{\phi}{2}+n \sin \frac{\phi}{2}\right)\right], \\
& \sigma_{y}^{I I}(x, y)=y \frac{a^{2} \tau_{\infty}}{|t|^{2} \sqrt{|t|}}\left(m \cos \frac{\phi}{2}+n \sin \frac{\phi}{2}\right), \\
& \tau_{x y}^{I I}(x, y)=\frac{\tau_{\infty}}{\sqrt{|t|}}\left[\left(x \cos \frac{\phi}{2}-y \sin \frac{\phi}{2}\right)+y \frac{a^{2}}{|t|^{2}}\left(m \sin \frac{\phi}{2}-n \cos \frac{\phi}{2}\right)\right],
\end{aligned}
$$

where the stress fields are expressed as a function of $x$ and $y$, with origin at the centre of the crack. The parameters $t, m, n$ and $\phi$ are defined as

$$
\begin{aligned}
t & =(x+i y)^{2}-a^{2}=\left(x^{2}-y^{2}-a^{2}\right)+i(2 x y)=m+i n, \\
m & =\operatorname{Re}(t)=\operatorname{Re}\left(z^{2}-a^{2}\right)=x^{2}-y^{2}-a^{2}, \\
n & =\operatorname{Im}(t)=\left(z^{2}-a^{2}\right)=2 x y, \\
\phi & =\operatorname{Arg}(\bar{t})=\operatorname{Arg}(m-i n) \quad \text { with } \phi \in[-\pi, \pi], i^{2}=-1 .
\end{aligned}
$$


For the problem analysed, the exact value of the SIF is given by

$$
K_{I, e x}=\sigma_{\infty} \sqrt{\pi a} \quad K_{I I, e x}=\tau_{\infty} \sqrt{\pi a} .
$$

Material parameters are Young's modulus $E=10^{7}$ and Poisson's ratio $\nu=0.333$. We consider loading conditions in pure mode I with $\sigma_{\infty}=100$ and $\tau_{\infty}=0$, and pure mode II with $\sigma_{\infty}=0$ and $\tau_{\infty}=100$. We assume plane strain conditions.

In the numerical analysis, we use a geometrical enrichment defined by a circular fixed enrichment area $B\left(x_{0}, r_{e}\right)$ with radius $r_{e}=2.5$, with its centre at the crack tip $x_{0}$ as proposed in [42]. Bilinear elements are considered in the models, using a sequence of uniformly refined meshes. For the numerical integration of standard elements we use a $2 \times 2$ Gaussian quadrature rule. We use a $5 \times 5$ quasipolar integration in the subdomains of the element containing the crack tip [42]. We do not consider correction for blending elements. Methods to address blending errors are proposed in $[43,44,45,46]$.

To evaluate the stress intensity factor $K$ we use an EDI technique [33]. For the primal problem we consider a square plateau function $q$ centred at the crack tip, as shown in Figure 3. $q=1$ for the domain defined by an inner square with side length 6 and $q=0$ for the part of the domain outside the outer square with side length $8, q$ is interpolated in-between the two squares. This plateau function is also used to define the subdomain $\Omega_{i}$ when extracting the quantity of interest in the dual problem. As the dual problem is also a singular problem we have to evaluate a second stress intensity factor. In this case, we use a plateau function such that $q=1$ for all nodes inside a square with side length 4.9 and $q=0$ otherwise.

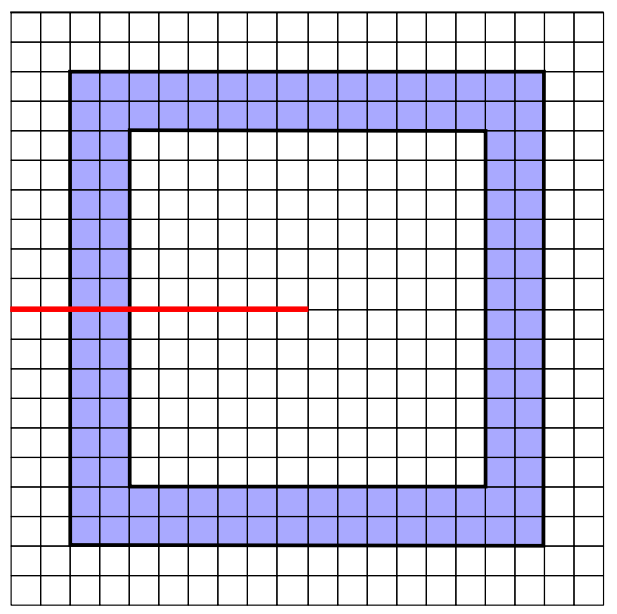

Figure 3: Domain of interest for the extraction of the stress intensity factor

In Figure 4 we represent the equivalent nodal forces used to solve the primal and dual problems. For the dual problem the vector of forces is constructed using the discrete approximation of the dual function. The Dirichlet boundary constraints are the same for both models. For the dual problem, we can see that the forces are distributed in the nodes located in the domain of interest. For the recovery of the primal and dual fields, we perform the splitting of stresses and enforce internal equilibrium, boundary equilibrium and the compatibility equation. 

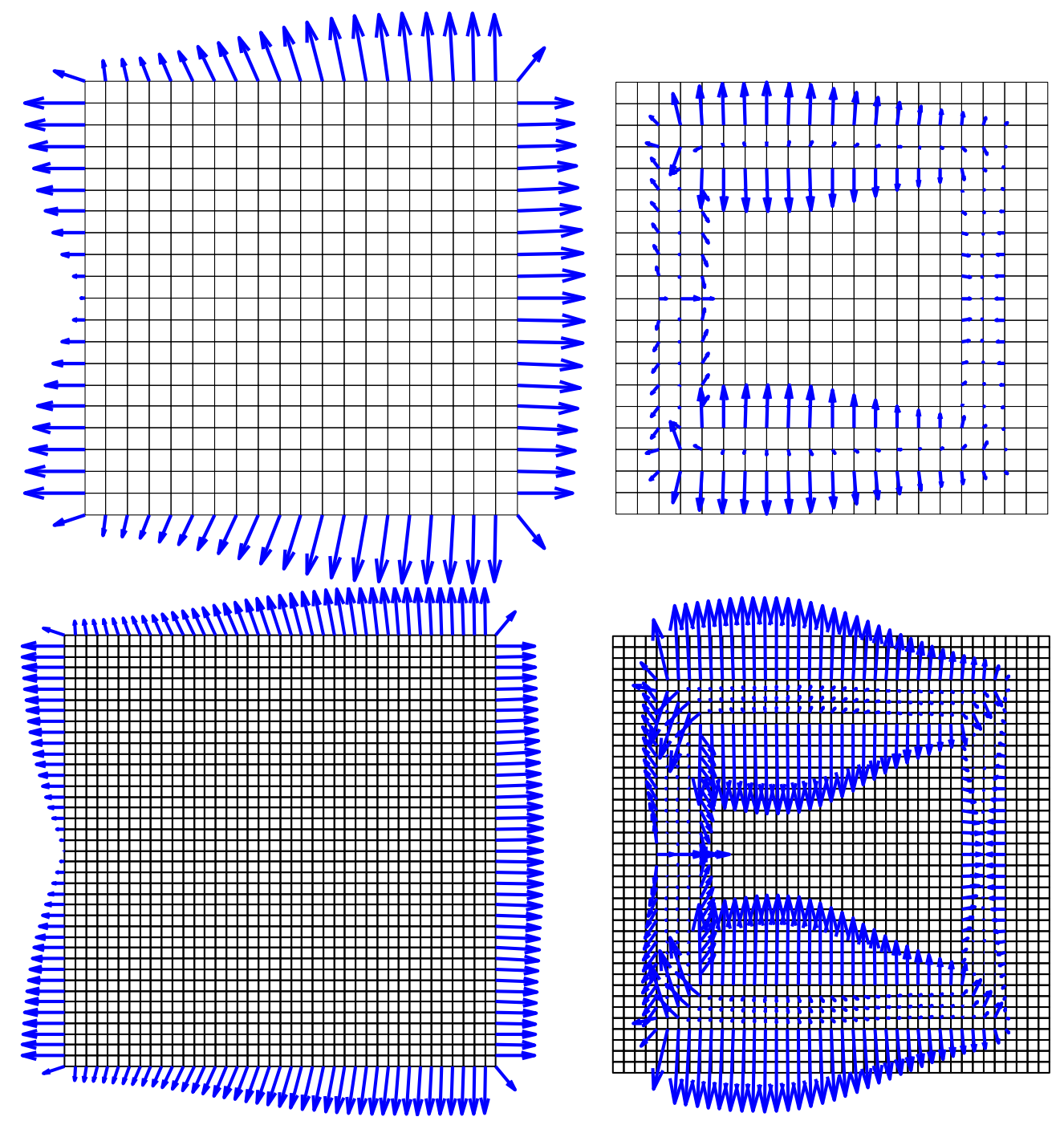

Figure 4: Equivalent forces at nodes for the primal (left) and dual (right) problems.

The yy-component of the stress field for the raw $\mathrm{FE}$ and the recovered solutions is represented in Figure 5. The enrichment area is indicated with a circle. In Figure 6 we show the same results for the dual problem. Notice how the recovery procedure smoothes the stresses along the interface of the domain of interest. As the dual problem is also characterised by the crack, we have to evaluate the corresponding stress intensity factor and perform the singular + smooth decomposition of the stress field.

Figure 7 shows the evolution of the effectivity index $\theta$ as we increase the number of degrees of freedom (dof). We consider as quantities of interest the two SIFs characterising two different loading conditions, i.e. mode I and mode II. We can see that for both quantities the error estimator yields effectivities close to the optimal value $\theta=1$.

In Tables 1 and 2 we indicate the values for the estimated, $\mathcal{E}$, and exact, $Q(\mathbf{e})$, errors, the global effectivity index $\theta$ and the effectivity for the quantity of interest $\theta_{\text {QoI }}$ using the proposed recovery technique and the standard SPR (denoted with $\dagger$ ). 

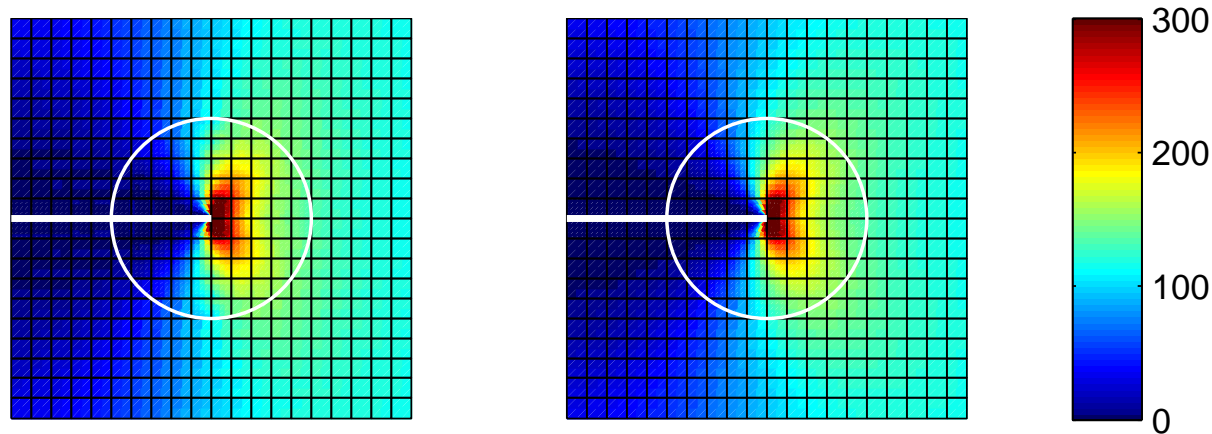

Figure 5: FE (left) and recovered (right) $\sigma_{y y}$ for the primal problem.
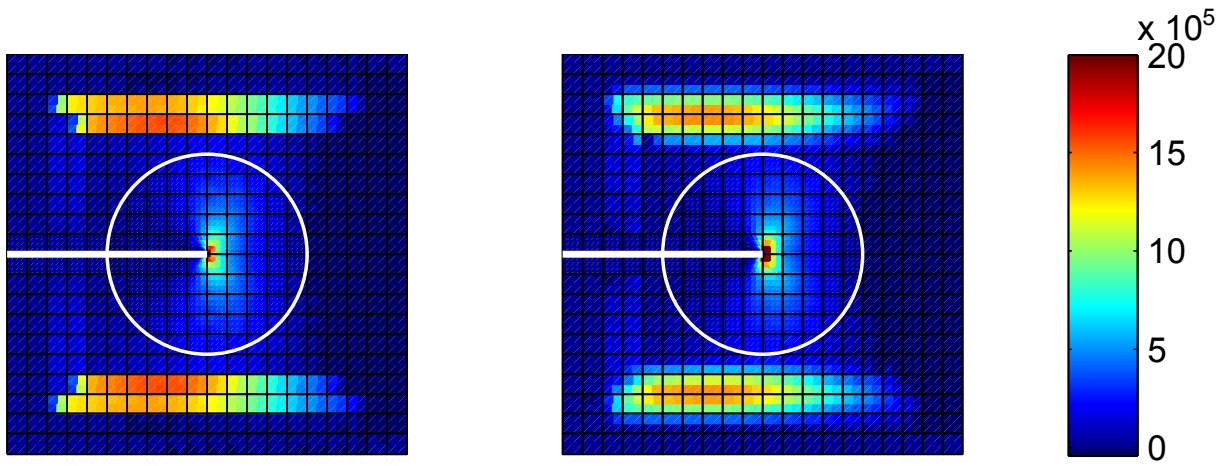

Figure 6: FE (left) and recovered fields (right) $\sigma_{y y}$ for the dual problem.

The magnitude of the exact error is accurately captured by the estimated obtained with the SPR-CX, which is clearly reflected in the good effectivity index for both loading modes. As expected, the effectivity in the quantities of interest $\theta_{Q o I}$ is highly accurate. For the SPR, although the value $\mathcal{E}$ decreases as we increase the number of dof, the error estimate is not as accurate as the estimate obtained with the SPR-CX and does not decrease as fast as the exact error, loosing asymptotic exactness. The SPR does not consider the splitting of the singular stresses, giving less accurate results close to the crack tip, and does not enforce equilibrium conditions in the primal and dual recovered fields, which results in a poorer description of the stresses close to the boundaries and the interface of the domain of interest.

Figure 8 compares the results of the proposed SPR-CX recovery with the standard SPR technique. In particular, the SPR cannot properly recover singular fields,

Table 1: Stress intensity factor $K_{\mathrm{I}}$ as QoI. ${ }^{\dagger}$ Results using the standard SPR recovery.

\begin{tabular}{rccccrrr}
\hline dof & $\mathcal{E}$ & $Q(\mathbf{e})$ & $\theta$ & $\theta_{Q o I}$ & $\mathcal{E}^{\dagger}$ & \multicolumn{1}{c}{$\theta^{\dagger}$} & $\theta_{Q o I}^{\dagger}$ \\
\hline 351 & 2.5264 & 2.1144 & 1.1948 & 1.00261 & 13.7089 & 6.4835 & 1.07333 \\
1,289 & 0.4822 & 0.5146 & 0.9369 & 0.99979 & 6.3880 & 12.4124 & 1.03715 \\
4,973 & 0.1140 & 0.1216 & 0.9376 & 0.99995 & 3.1756 & 26.1141 & 1.01932 \\
19,637 & 0.0267 & 0.0278 & 0.9617 & 0.99999 & 1.5789 & 56.7646 & 1.00981 \\
\hline
\end{tabular}


$\theta$

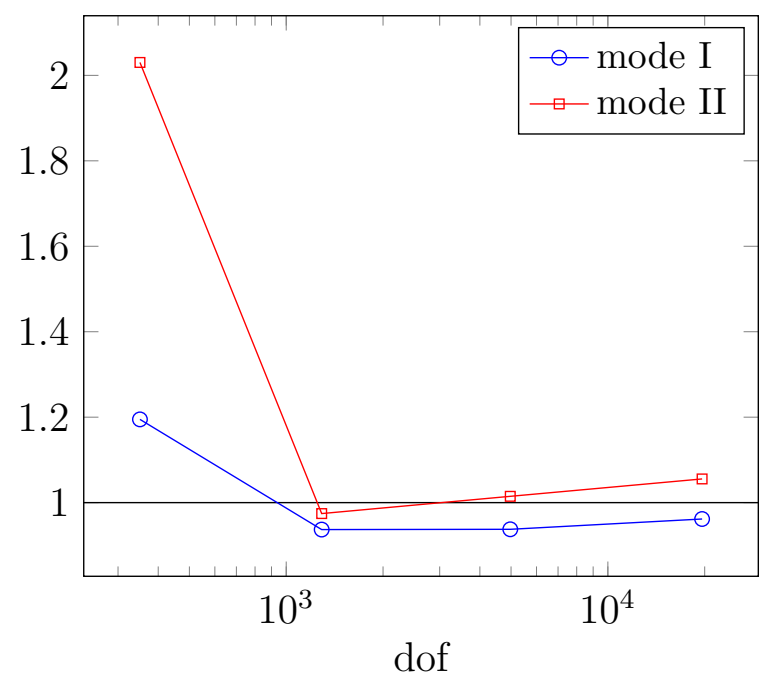

Figure 7: Evolution of the effectivity index $\theta$ considering the SIF as quantity of interest under mode I and mode II loading conditions.

Table 2: Stress intensity factor $K_{\mathrm{II}}$ as QoI. ${ }^{\dagger}$ Results using the standard SPR recovery.

\begin{tabular}{rccccccc}
\hline dof & $\mathcal{E}$ & $Q(\mathbf{e})$ & $\theta$ & $\theta_{\text {QoI }}$ & $\mathcal{E}^{\dagger}$ & $\theta^{\dagger}$ & $\theta_{\text {QoI }}^{\dagger}$ \\
\hline 351 & 2.2886 & 1.1273 & 2.0302 & 1.00735 & 5.6052 & 4.9724 & 1.02832 \\
1,289 & 0.2827 & 0.2902 & 0.9744 & 0.99995 & 2.2203 & 7.6517 & 1.01221 \\
4,973 & 0.0700 & 0.0689 & 1.0147 & 1.00001 & 1.1078 & 16.0677 & 1.00657 \\
19,637 & 0.0168 & 0.0159 & 1.0554 & 1.00001 & 0.5502 & 34.6562 & 1.00338 \\
\hline
\end{tabular}

thus, the error estimate provided by the technique does not converge to the exact error [32]. This behaviour is similar for the two loading modes.

In Figure 9 we represent the distribution of the estimated error for the second mesh of the sequence for the error in energy norm $\left\|\mathbf{e}_{e s}\right\|$ and the error considering the quantity of interest $\mathcal{E}$. This error distribution might guide the refinement during the adaptivity procedure. The approach based on energy norm estimates that the most critical part is located in the vicinity of the singular point whilst the goal oriented approach also considers the domain where the information of the QoI is extracted.

\section{Conclusions and future work.}

We have presented a locally equilibrated recovery procedure for goal oriented error estimation in XFEM. We have considered as the design parameter the generalised stress intensity factor that characterises the solution of singular problems in the context of linear elastic fracture mechanics. The technique proposes the use of a stress recovery that provides locally equilibrated stress fields for both the primal and the dual problem.

To formulate the dual problem we consider the linear equivalent domain inte- 
$\theta$

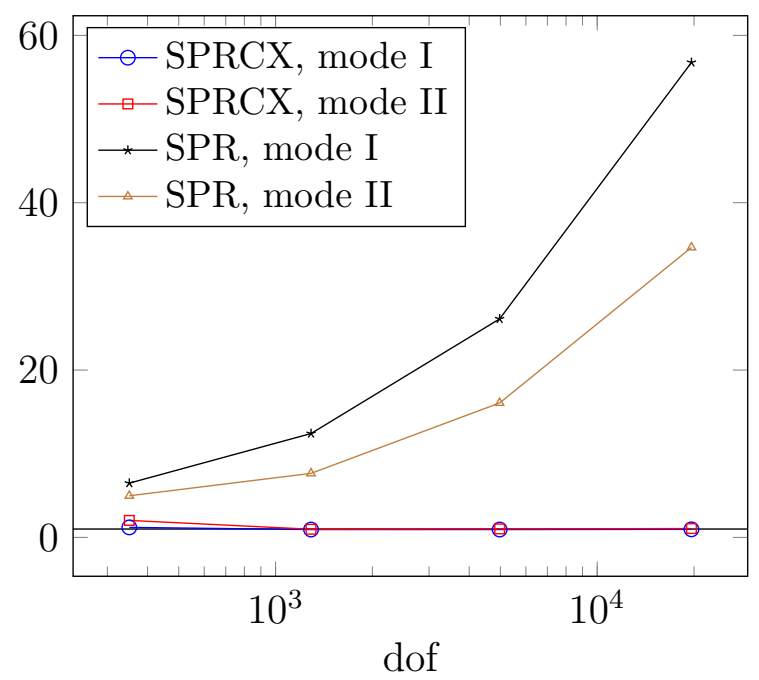

Figure 8: Evolution of the effectivity index $\theta$ for the SPRCX and SPR. Mode I and mode II loading conditions.

gral representing $K$ to obtain the applied loads of the dual FE approximation. To perform the recovery of the primal and dual solutions we consider three main ideas: (i) enforcement of the internal equilibrium equation, (ii) enforcement of boundary equilibrium and (iii) splitting of the stress field into singular and smooth parts.

The proposed technique has been tested with problems under different loading conditions. The obtained results show that the error estimator accurately captures the exact error in the evaluation of the stress intensity factor.

\section{Acknowledgements}

This work was supported by the EPSRC grant EP/G042705/1 "Increased Reliability for Industrially Relevant Automatic Crack Growth Simulation with the eXtended Finite Element Method". Stéphane Bordas also thanks partial funding for his time provided by the European Research Council Starting Independent Research Grant (ERC Stg grant agreement No. 279578) "RealTCut Towards real time multiscale simulation of cutting in non-linear materials with applications to surgical simulation and computer guided surgery". This work has been carried out within the framework of the research project DPI2010-20542 of the Ministerio de Ciencia e Innovación (Spain). The financial support of the FPU program (AP2008-01086), the funding from Universitat Politècnica de València and Generalitat Valenciana (PROMETEO/2012/023) are also acknowledged. 

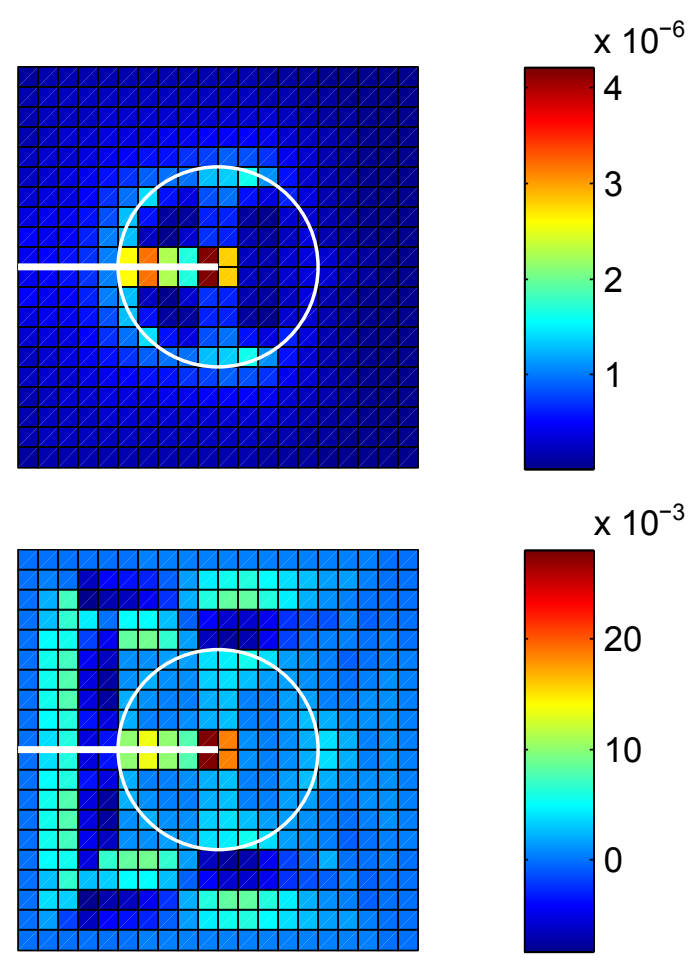

Figure 9: Distribution of the estimated error considering the error in energy norm (top) and the error in the quantity of interest $\mathcal{E}$ (bottom). 


\section{References}

[1] Anderson TL. Fracture Mechanics: Fundamentals and Applications, vol. 2. CRC Press: Boca Ratón, Florida, 1995.

[2] Gross D, Seelig T. Fracture mechanics with an introduction to micromechanics. 2nd edn., Springer, 2011.

[3] Rüter M, Stein E. Goal-oriented a posteriori error estimates in linear elastic fracture mechanics. Computer Methods in Applied Mechanics and Engineering Jan 2006; 195(4-6):251-278.

[4] Panetier J, Ladevèze P, Chamoin L. Strict and effective bounds in goal-oriented error estimation applied to fracture mechanics problems solved with XFEM. International Journal for Numerical Methods in Engineering 2010; 81(6):671700 .

[5] Babuška I, Rheinboldt WC. A-posteriori error estimates for the finite element method. International Journal for Numerical Methods in Engineering 1978; 12(10):1597-1615.

[6] Zienkiewicz OC, Zhu JZ. A simple error estimator and adaptive procedure for practical engineering analysis. International Journal for Numerical Methods in Engineering 1987; 24(2):337-357.

[7] Ladevèze P, Leguillon D. Error estimate procedure in the finite element method and applications. SIAM Journal on Numerical Analysis 1983; 20(3):485-509.

[8] Almeida Pereira OJB, Moitinho de Almeida JP, Maunder EAW. Adaptive methods for hybrid equilibrium finite element models. Computer Methods in Applied Mechanics and Engineering Jul 1999; 176(1-4):19-39.

[9] Babuška I, Strouboulis T, Upadhyay CS. A model study of the quality of a posteriori error estimators for linear elliptic problems. Error estimation in the interior of patchwise uniform grids of triangles. Computer Methods in Applied Mechanics and Engineering 1994; 114(3-4):307-378.

[10] Babuška I, Strouboulis T, Upadhyay CS, Gangaraj SK, Copps K. Validation of a posteriori error estimators by numerical approach. International Journal for Numerical Methods in Engineering Apr 1994; 37(7):1073-1123.

[11] Babuška I, Strouboulis T, Upadhyay CS. A model study of the quality of a posteriori error estimators for finite element solutions of linear elliptic problems, with particular reference to the behaviour near the boundary. International Journal for Numerical Methods in Engineering 1997; 40(14):2521-2577.

[12] Zienkiewicz OC, Zhu JZ. The superconvergent patch recovery and a posteriori error estimates. Part 1: The recovery technique. International Journal for Numerical Methods in Engineering 1992; 33(7):1331-1364. 
[13] Ainsworth M, Oden JT. A posteriori Error Estimation in Finite Element Analysis. John Wiley \& Sons: Chichester, 2000.

[14] Paraschivoiu M, Peraire J, Patera AT. A posteriori finite element bounds for linear-functional outputs of elliptic partial differential equations. Computer Methods in Applied Mechanics and Engineering 1997; 150(1-4):289-312.

[15] Ladevèze P, Rougeot P, Blanchard P, Moreau JP. Local error estimators for finite element linear analysis. Computer Methods in Applied Mechanics and Engineering 1999; 176(1-4):231-246.

[16] Oden JT, Prudhomme S. Goal-oriented error estimation and adaptivity for the finite element method. Computers 85 Mathematics with Applications Mar 2001; 41(5-6):735-756.

[17] Cirak F, Ramm E. A posteriori error estimation and adaptivity for linear elasticity using the reciprocal theorem. Computer Methods in Applied Mechanics and Engineering Apr 1998; 156(1-4):351-362.

[18] Moitinho de Almeida JP, Almeida Pereira OJB. Upper bounds of the error in local quantities using equilibrated and compatible finite element solutions for linear elastic problems. Computer Methods in Applied Mechanics and Engineering Jan 2006; 195(4-6):279-296.

[19] González-Estrada OA, Nadal E, Ródenas JJ, Kerfriden P, Bordas SPA, Fuenmayor FJ. Error estimation in quantities of interest by locally equilibrated superconvergent patch recovery. Computational Mechanics 2013; (submitted):128.

[20] Moës N, Dolbow J, Belytschko T. A finite element method for crack growth without remeshing. International Journal for Numerical Methods in Engineering Sep 1999; 46(1):131-150.

[21] Amani J, Saboor Bagherzadeh A, Rabczuk T. Error estimate and adaptive refinement in mixed discrete least squares meshless method. Mathematical Problems in Engineering 2014; 2014:1-16.

[22] Ghorashi S, Valizadeh N, Mohammadi S. Extended isogeometric analysis for simulation of stationary and propagating cracks. International Journal for $\mathrm{Nu}$ merical Methods in Engineering 2012; 89:1069-1101.

[23] Bordas SPA, Duflot M. Derivative recovery and a posteriori error estimate for extended finite elements. Computer Methods in Applied Mechanics and Engineering 2007; 196(35-36):3381-3399.

[24] Duflot M, Bordas SPA. A posteriori error estimation for extended finite elements by an extended global recovery. International Journal for Numerical Methods in Engineering 2008; 76:1123-1138. 
[25] Ródenas JJ, González-Estrada OA, Tarancón JE, Fuenmayor FJ. A recoverytype error estimator for the extended finite element method based on singular+smooth stress field splitting. International Journal for Numerical Methods in Engineering 2008; 76(4):545-571.

[26] Ródenas JJ, González-Estrada OA, Díez P, Fuenmayor FJ. Accurate recoverybased upper error bounds for the extended finite element framework. Computer Methods in Applied Mechanics and Engineering 2010; 199(37-40):2607-2621.

[27] Strouboulis T, Zhang L, Wang D, Babuška I. A posteriori error estimation for generalized finite element methods. Computer Methods in Applied Mechanics and Engineering 2006; 195(9-12):852-879.

[28] Gerasimov T, Rüter M, Stein E. An explicit residual-type error estimator for Q 1 -quadrilateral extended finite element method in two-dimensional linear elastic fracture mechanics. International Journal for Numerical Methods in Engineering 2012; 90(April):1118-1155.

[29] Panetier J, Ladevèze P, Louf F. Strict bounds for computed stress intensity factors. Computers \& Structures Aug 2009; 87(15-16):1015-1021.

[30] Rüter M, Gerasimov T, Stein E. Goal-oriented explicit residual-type error estimates in XFEM. Computational Mechanics 2013; 52(2):361-376.

[31] Pannachet T, Sluys LJ, Askes H. Error estimation and adaptivity for discontinuous failure. International Journal for Numerical Methods in Engineering $2009 ; \mathbf{7 8}(5): 528-563$.

[32] González-Estrada OA, Ródenas JJ, Bordas SPA, Duflot M, Kerfriden P, Giner E. On the role of enrichment and statical admissibility of recovered fields in a-posteriori error estimation for enriched finite element methods. Engineering Computations 2012; 29(8):1-28.

[33] Szabó BA, Babuška I. Finite Element Analysis. John Wiley \& Sons: New York, 1991.

[34] Ródenas JJ. Goal Oriented Adaptivity: Una introducción a través del problema elástico lineal. Technical Report, CIMNE, PI274, Barcelona, Spain 2005.

[35] González-Estrada OA, Ródenas JJ, Nadal E, Bordas SPA, Kerfriden P. Equilibrated patch recovery for accurate evaluation of upper error bounds in quantities of interest. Adaptive Modeling and Simulation. Proceedings of $V$ ADMOS 2011, Aubry D, Díez P, Tie B, Parés N (eds.), CINME: Paris, 2011.

[36] Verdugo F, Díez P, Casadei F. Natural quantities of interest in linear elastodynamics for goal oriented error estimation and adaptivity. Adaptive Modeling and Simulation. Proceedings of V ADMOS 2011, Aubry D, Díez P, Tie B, Parés N (eds.), CIMNE: Paris, 2011. 
[37] Díez P, Ródenas JJ, Zienkiewicz OC. Equilibrated patch recovery error estimates: simple and accurate upper bounds of the error. International Journal for Numerical Methods in Engineering 2007; 69(10):2075-2098.

[38] Ródenas JJ, Tur M, Fuenmayor FJ, Vercher A. Improvement of the superconvergent patch recovery technique by the use of constraint equations: the SPR-C technique. International Journal for Numerical Methods in Engineering 2007; 70(6):705-727.

[39] Giner E, Tur M, Fuenmayor FJ. A domain integral for the calculation of generalized stress intensity factors in sliding complete contacts. International Journal of Solids and Structures Feb 2009; 46(3-4):938-951.

[40] Blacker T, Belytschko T. Superconvergent patch recovery with equilibrium and conjoint interpolant enhancements. International Journal for Numerical Methods in Engineering 1994; 37(3):517-536.

[41] Giner E, Fuenmayor FJ, Baeza L, Tarancón JE. Error estimation for the finite element evaluation of GI and GII in mixed-mode linear elastic fracture mechanics. Finite Elements in Analysis and Design 2005; 41(11-12):1079-1104.

[42] Béchet E, Minnebo H, Moës N, Burgardt B. Improved implementation and robustness study of the X-FEM for stress analysis around cracks. International Journal for Numerical Methods in Engineering Oct 2005; 64(8):1033-1056.

[43] Chessa J, Wang H, Belytschko T. On the construction of blending elements for local partition of unity enriched finite elements. International Journal for Numerical Methods in Engineering Jun 2003; 57(7):1015-1038.

[44] Gracie R, Wang H, Belytschko T. Blending in the extended finite element method by discontinuous Galerkin and assumed strain methods. International Journal for Numerical Methods in Engineering 2008; 74(11):1645-1669.

[45] Fries T. A corrected XFEM approximation without problems in blending elements. International Journal for Numerical Methods in Engineering 2008; 75(5):503-532.

[46] Tarancón JE, Vercher A, Giner E, Fuenmayor FJ. Enhanced blending elements for XFEM applied to linear elastic fracture mechanics. International Journal for Numerical Methods in Engineering 2009; 77(1):126-148. 